\title{
Getting to the bottom of top
}

The announcement of the probable discovery of the top quark at Fermilab in the United States is a landmark in the history of particle physics, but an expected one. It would be enlivening if the next were unexpected.

THE top quark may have been discovered at last, at Fermilab, the high-energy physics laboratory near Chicago. Last week, those attending the annual meeting of the American Physical Society at Arlington, Virginia waited in vain for a statement. But this week Fermilab announced - with proper caution - that it believes it has manufactured a handful of top quarks. The mass of the particle appears to be within 10 per cent of $174 \mathrm{GeV}$, within recently established upper and lower bounds, but higher than many had expected.

As things stand, Fermilab is the only laboratory in the world equipped to announce the discovery of the top quark; until CERN has its Large Hadron Collider running, no other place can hope to get within range.

But why all the excitement about the top quark, of whose ultimate existence nobody has much doubt? It has something to do with the principle that seeing is believing; inference, however neat the underlying theory, is always less persuasive than demonstration. But it would be also be galling for highenergy physicists to be basing calculations of the properties of particles on the assumption that the top quark exists when they had not been able to manufacture a single specimen.

Quarks are, of course, the ingredients of particles of nuclear matter such as protons and neutrons. In the standard model, they come in pairs, one pair for each of the three 'generations' that make up the family of electron-like particles, and have amusing names - 'up' and 'down', 'strange' and 'charm', 'bottom' and ...'top', of course. Quarks cannot exist in isolation, but only in pairs (when they make mesons) or triplets (when they make nucleons).

Fermilab is wise to have been cautious. Ten years ago, the UA1 collaboration at CERN had a handful of events that seemed tantalizingly as if caused by the production and decay of top quarks. A lengthy Physics Letter (147B, 493; 1984) assessed the pros and cons and concluded that, if the events were due to the decay of top quarks, their mass must be between 30 and $50 \mathrm{GeV}$.But as more data accumulated, the background noise turned out to be larger than originally measured and

Frank Close is at the Rutherford Appleton Laboratory, Chilton, Didcot, Oxfordshire OX11 0QX, UK

NATURE · VOL 368 • 28 APRIL 1994 the handful of particles ceased to be significant.

Since then, experiments at CERN and at Fermilab have gradually pushed up the lower bound on the top quark's mass to a value of $\geq 131 \mathrm{GeV}$, (S. Abachi et al. Phys. Rev. Lett. 72, 2138; 1994). That lower limit is surprisingly high, some 50 per cent more than the mass of the intermediate vector boson called the $Z^{0}$ and even greater than the mass of the atomic nucleus of a silver atom.

For the time being, Fermilab is the only accelerator that could directly produce top quarks with such great masses. There are two experimental teams called CDF (for Collider Detector at Fermilab) and prosaically D0, each with hundreds of members. Reaching an agreed position on the interpretation of data, and maintaining prudent or seemly silence in the process, is fraught with problems, especially when collaborators are analysing data at institutions on three continents. And everybody is conscious of the dangers of premature claims that turn out to be false, of which there has already been a sprinkling.

Since mid-March, rumour has nevertheless been rife that Fermilab was on to something. If nothing else, graduate students have been turning in theses with samples of the data gathered at Chicago. But the barely suppressed excitement of the people there has also been significant.

So it is worth remembering that there is already good evidence for the existence of the top quark. If it is accepted (and there is no choice) that quantum field theory applies to quarks as it does to electrons, then the top quark has already manifested itself in its perturbation of precisely observable quantities at CERN's LEP, much as fluctuations of the quantum electromagnetic field determine the Lamb shift in the hyperfine spectra of atoms.

For what it is worth, the bosons $Z^{0}$ and $\mathrm{W}^{ \pm}$were similarly inferred from precision data before their production at CERN. But the data so far collected at LEP with a bearing on the top quark provide only an upper limit of $200 \mathrm{GeV}$ for its mass.

It is natural that particle physicists seek direct materialization of top quarks or their composites with other quarks, for the signal of top decay is expected not to be simply diagnostic. Among other pathways, for example, the decay of top is expected to yield a W and a bottom quark, each of which will then further decay into final states, electrons and muons for example. But there are other processes, unrelated to top quarks, that can yield the same signals. Isolating the signal from the noise is the essence of the hunt for the top quark. And the more massive it is, the more difficult it must be to separate the signal from the background.

The CDF collaboration's draft paper has the positive title "Evidence for top quark production in $\bar{p}$ collisions at $1.8 \mathrm{TeV}$ " and runs to nearly 200 pages. The evidence consists of a handful of possible 'signal' events with the characteristics of the top quark, but there are other features that do not fit exactly into the expectations, and which the paper recognizes and debates. The events, assumed real, correspond to

$$
m_{\mathrm{t}}=174 \pm 10 \pm \frac{13}{12} \mathrm{GeV} \text {. }
$$

The statistical significance is low enough that one must allow for the possibility that subsequent data may home in on a different mass and that these events will be assigned to background or to some other phenomenon. It is a powerful measure of the caution with which the result is put forward that those concerned have written 200 pages of a full paper before boiling this down to what is expected also to be a Letter (in Physical Review Letters). The data are what the title says: evidence (but not proof) that the top quark exists.

How, if at all, will this development change high-energy physics? When the top is eventually agreed upon, it will indeed provide the final ingredient of the third generation of matter. High-energy physics is also awaiting the Higgs boson, and there are questions such as that of 'supersymmetry' still to be decided. They may be tasks for the Large Hadron Collider, if and when that is built at CERN.

Meanwhile, we should not overlook the possibility of being surprised. When 'strange' matter was first found in 1947, the concept of a quark had not been formulated. And the signal that led to the recognition of the tau lepton (heavy cousin of the electron and the muon) was extracted from noisy data only with dedication comparable with that which has probably now given us the top quark. "Who ordered that?", people were asking nearly twenty years ago. The same cry may yet be heard at the accelerator laboratories in the years ahead.

Frank Close \& John Maddox 